\title{
Effects of weight loss and exercise on insulin resistance, and intramyocellular triacylglycerol, diacylglycerol and ceramide
}

\author{
J. J. Dubé • F. Amati • F. G. S. Toledo • \\ M. Stefanovic-Racic • A. Rossi • P. Coen • \\ B. H. Goodpaster
}

Received: 17 August 2010 / Accepted: 10 January 2011 /Published online: 17 February 2011

(C) Springer-Verlag 2011

\begin{abstract}
Aims/hypothesis Intramyocellular lipids, including diacylglycerol (DAG) and ceramides, have been linked to insulin resistance. This randomised repeated-measures study examined the effects of diet-induced weight loss (DIWL) and aerobic exercise (EX) on insulin sensitivity and intramyocellular triacylglycerol (IMTG), DAG and ceramide. Methods Sixteen overweight to obese adults (BMI 30.6 \pm 0.8 ; $67.2 \pm 4.0$ years of age) with either impaired fasting glucose, or impaired glucose tolerance completed one of two lifestyle interventions: DIWL $(n=8)$ or $\mathrm{EX}(n=8)$. Insulin sensitivity was determined using hyperinsulinaemic-euglycaemic clamps. Intramyocellular lipids were measured in muscle biopsies using histochemistry and tandem mass spectrometry. Results Insulin sensitivity was improved with DIWL (20.6 $4.7 \%)$ and EX (19.2 $\pm 12.9 \%)$. Body weight and body fat were
\end{abstract}

Electronic supplementary material The online version of this article (doi:10.1007/s00125-011-2065-0) contains supplementary material, which is available to authorised users.

J. J. Dubé $\cdot$ F. Amati · F. G. S. Toledo $\cdot$ M. Stefanovic-Racic $\cdot$

P. Coen $\cdot$ B. H. Goodpaster $(\square)$

Division of Endocrinology and Metabolism,

Department of Medicine,

University of Pittsburgh School of Medicine,

University of Pittsburgh,

3459 Fifth Avenue, MUH N807,

Pittsburgh, PA 15213, USA

e-mail: bgood@pitt.edu

A. Rossi

Department of Geriatrics, University of Verona,

Verona, Italy

P. Coen

Department of Health and Physical Activity,

University of Pittsburgh,

Pittsburgh, PA, USA decreased by both interventions, with greater decreases in DIWL compared with EX. Muscle glycogen, IMTG content and oxidative capacity were all significantly $(p<0.05)$ decreased with DIWL and increased with EX. There were decreases in DAG with DIWL $(-12.4 \pm 14.6 \%)$ and EX $(-40.9 \pm 12.0 \%)$. Ceramide decreased with EX $(-33.7 \pm$ $11.2 \%)$, but not with DIWL. Dihydroceramide was decreased with both interventions. Sphingosine was decreased only with EX. Changes in total DAG, total ceramides and other sphingolipids did not correlate with changes in glucose disposal. Stearoyl-coenzyme A desaturase 1 (SCD1) content was decreased with DIWL $(-19.5 \pm$ $8.5 \%, p<0.05)$, but increased with EX $(19.6 \pm 7.4 \%$, $p<0.05$ ). Diacylglycerol acyltransferase 1 (DGAT1) was unchanged with the interventions.

Conclusions/interpretation Diet-induced weight loss and exercise training both improved insulin resistance and decreased DAG, while only exercise decreased ceramides, despite the interventions having different effects on IMTG. These alterations may be mediated through differential changes in skeletal muscle capacity for oxidation and triacylglycerol synthesis.

Trial registration: ClinicalTrials.gov NCT00766298

Funding: ADA Clinical Research Award (B. H. Goodpaster), NIH R01 AG20128 (B. H. Goodpaster), Obesity and Nutrition Research (1P30DK46204) and Clinical and Translational Research Centers (UL1 RR024153)

Keywords Ceramide - Diacylglycerol - Insulin resistance · Skeletal muscle
Abbreviations
CTRC Clinical Translational Research Center
DAG Diacylglycerol 


$\begin{array}{ll}\text { DGAT1 } & \text { Diacylglycerol acyltransferase 1 } \\ \text { DIWL } & \text { Diet-induced weight loss (intervention) } \\ \text { EX } & \text { Aerobic exercise (intervention) } \\ \text { FFM } & \text { Fat-free mass } \\ \text { IGT } & \text { Impaired glucose tolerance } \\ \text { IMCL } & \text { Intramyocellular lipid } \\ \text { IMTG } & \text { Intramyocellular triacylglycerol } \\ \text { NGT } & \text { Normal glucose tolerance } \\ \text { PKC } & \text { Protein kinase C } \\ \text { SCD1 } & \text { Stearoyl-coenzyme A desaturase 1 } \\ \text { SDH } & \text { Succinate dehydrogenase }\end{array}$

\section{Introduction}

Skeletal muscle insulin resistance is a key feature in type 2 diabetes mellitus and in individuals at high risk for the development of diabetes [1]. Obesity and physical inactivity are major contributors to insulin resistance [2], although the exact mechanisms by which they cause insulin resistance are unknown. Over the last decade there has been considerable interest in the role of intramyocellular lipids (IMCLs) in insulin resistance. Early evidence from cell culture [3], animal models [4] and human studies [5] strongly suggested that accumulation of intramyocellular triacylglycerol (IMTG) was responsible for insulin resistance of obesity and type 2 diabetes. We found that diet-induced weight loss (DIWL) decreased IMCL in obese individuals with and without type 2 diabetes [6] in conjunction with enhanced insulin sensitivity [7]. We subsequently reported an 'athletes' paradox' in which endurance-trained athletes are markedly insulin sensitive despite having high IMCL [8], a phenomenon we $[9,10]$ and others $[11,12]$ have confirmed with exercise training interventions. Accumulation of IMCL within the context of improved insulin sensitivity may be mediated through the lipogenic enzymes stearoyl-coenzyme A desaturase 1 (SCD1) [13, 14] and diacylglycerol acyltransferase 1 (DGAT1) [15]. Thus, although weight loss and exercise can both improve insulin sensitivity [7, 16] they may have distinctly different effects on IMCL [6, 9]. This hypothesis has not been directly tested in a randomised trial.

IMCL may represent a surrogate for other potentially lipotoxic metabolites such as diacylglycerol (DAG) and/or ceramide which, in cell culture systems and animal models, have been demonstrated to directly mediate skeletal muscle insulin action [17]. The mechanisms linking skeletal muscle DAG and ceramide accumulation and insulin resistance involve activation of various isoforms of protein kinase $\mathrm{C}$ (PKC) $[18,19]$ or inhibition of protein kinase B signalling [20]. Hoy et al. [21] have recently challenged the notion that either DAG or ceramide within skeletal muscle is linked with insulin resistance. Following $5 \mathrm{~h}$ of lipid oversupply in rodents, there were no changes in either DAG or ceramide content, yet several components of the insulin-signalling cascade were repressed. Data linking DAG or ceramide to human skeletal muscle insulin resistance are conflicting: while some studies have shown that DAG [22] and ceramide [18] are related to obesity or insulin resistance [23], others have failed to observe these associations $[24,25]$. The complexity of these lipids, including their fatty acid composition, is one potentially key aspect underlying these inconsistencies that has not been adequately explored.

Human intervention studies designed to improve insulin sensitivity could provide the next level of evidence concerning whether DAG or ceramide play a role in skeletal muscle insulin resistance. Few such studies have been conducted [26]. While we [9] and others [26] have recently reported that exercise training improves insulin sensitivity and decreases both total ceramide and DAG in humans, direct comparisons of the effects of exercise and DIWL on insulin sensitivity, IMCL, DAG, ceramide and other sphingolipids have not been conducted. Determining intervention effects on the subspecies profile of these complex lipids using quantitative mass spectrometry could yield novel information about their role in insulin resistance. We tested the primary hypothesis that, despite opposing effects on IMCL, exercise and DIWL would both decrease DAG and ceramide content within skeletal muscle concomitant with improved insulin sensitivity in overweight sedentary adults. This study provides novel insight into both the potential role of these complex lipids in human insulin resistance and whether they may be modifiable targets to prevent or treat insulin resistance associated with obesity, ageing or type 2 diabetes.

\section{Methods}

Study participants

Men and women aged 60-75 years were recruited though advertisements in the Pittsburgh area. Volunteers were eligible for participation if they were sedentary by self-report (structured exercise $\leq 1$ day per week), weight stable $(< \pm 3 \mathrm{~kg}$ in the previous 6 months), overweight to obese (BMI, 25.0 $38.0 \mathrm{~kg} / \mathrm{m}^{2}$ ) and a non-smoker. Upon further medical screening at the Clinical Translational Research Center (CTRC), volunteers with uncontrolled hypertension (blood pressure $>150 \mathrm{mmHg}[20 \mathrm{kPa}]$ systolic and $>95 \mathrm{mmHg}$ [12.7 $\mathrm{kPa}]$ diastolic), anaemia (haematocrit $<34 \%$ ), elevated liver enzymes ( $25 \%$ above normal), proteinuria 
or hypothyroidism (sensitive thyroid-stimulating hormone $>8$ ) were excluded. Individuals taking chronic medications known to adversely affect glucose homeostasis were excluded. Individuals using anti-hypertensive $(n=7)$ and lipid-lowering medications $(n=6)$ were included and continued their pharmacotherapy throughout the study. Participants with ECG abnormalities observed during the maximal aerobic capacity test or a history of unstable coronary artery disease were excluded.

Following the medical screen, volunteers completed a $2 \mathrm{~h}$ 75 g OGTT. Volunteers were classified as: normal fasting glucose $(<5.5 \mathrm{mmol} / \mathrm{l})$ or impaired fasting glucose ( $\geq 5.5 \mathrm{mmol} / \mathrm{l}$ ); normal glucose tolerance (NGT, $n=6 ; 2 \mathrm{~h}$ OGTT plasma glucose $<7.0 \mathrm{mmol} / \mathrm{l}$ ), or impaired glucose tolerance (IGT, $n=15 ; 2$ h OGTT plasma glucose $>7.8 \mathrm{mmol} / \mathrm{l}$ ). Participants provided written consent to the protocol approved by the University of Pittsburgh's Institutional Review Board.

\section{Study groups}

Volunteers with normal fasting glucose or impaired fasting glucose were randomised into one of two 16 week interventions: DIWL or aerobic exercise (EX). The distribution of glucose tolerance across intervention groups was: DIWL (IGT $n=8)$ and EX (IGT $n=4$ and NGT $n=4)$. Data are reported for the 16 volunteers $(\mathrm{DIWL}=8$ and $\mathrm{EX}=8$ ) who completed the interventions. The dropout rate was $\mathrm{DIWL}=\sim 10 \%$ and $\mathrm{EX}=\sim 10 \%$ (Fig. 1).

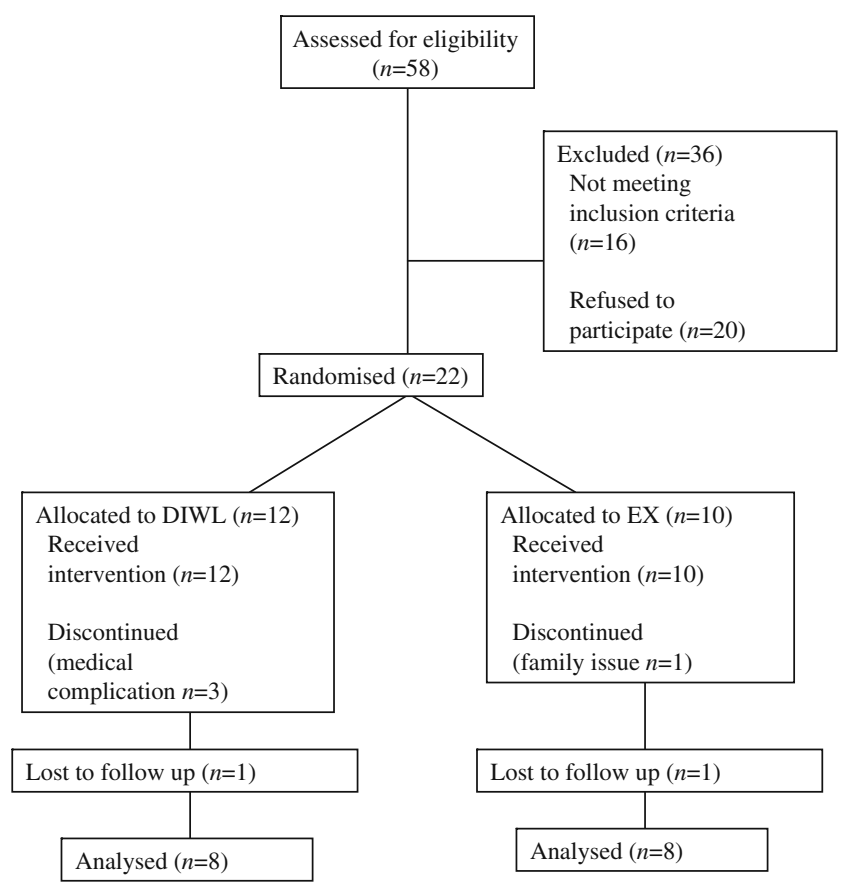

Fig. 1 Patient flow diagram
Diet-induced weight loss

The goal of this intervention was to achieve a $10 \%$ weight loss; a reduction of 2,093.4-4,186.8 $\mathrm{kJ} /$ day (based on recent food records/history) and low-fat ( $<30 \%$ of energy from fat) diet. A registered dietitian administered the weightloss programme individually to participants and provided a weekly dietary prescription. Individuals were kept relatively weight stable for the last 2 weeks of the 16 week period $(-0.54 \pm 0.79 \mathrm{~kg})$ to account for potential effects of acute negative energy balance on key outcome variables.

Aerobic exercise

Participants progressed to 4-5 days/week, $45 \mathrm{~min} / \mathrm{session}$ $(\sim 180 \mathrm{~min} /$ week) of moderate-intensity (determined by heart rate or perceived exertion [10]) supervised and unsupervised exercise (mostly stationary cycling and some walking) for 16 weeks. The heart rate was recorded (Polar Electro Oy, Kempele, Finland) during the exercise sessions to monitor exercise intensity and total volume. Participants were instructed to maintain current diet throughout their intervention. They met with the registered dietitian biweekly for body weight assessment and dietary intake review.

Insulin sensitivity

Individuals were admitted to the CTRC on the evening before the glucose clamp, fed a standardised meal, and remained fasted overnight. The next morning ( 07:00 hours), insulin sensitivity was measured as rates of peripheral insulinstimulated glucose disposal $\left(R_{\mathrm{d}}\right)$ during a $4 \mathrm{~h}$ hyperinsulinaemic-euglycaemic clamp (hyperinsulinaemic phase at $40 \mathrm{mU} \mathrm{m}^{-2} \min ^{-1}$ ) [7]. To measure $R_{\mathrm{d}}$ and residual endogenous glucose production, a primed $(0.22 \mu \mathrm{mol}$ $\left.1^{-1} \mathrm{~kg}^{-1}\right)$, continuous $\left(17.6 \mu \mathrm{mol} 1^{-1} \mathrm{~kg}^{-1} \mathrm{~min}^{-1}\right)$ infusion of $\left[6,6-{ }^{2} \mathrm{H}_{2}\right]$ glucose was given. $\left[6,6-{ }^{2} \mathrm{H}_{2}\right]$ glucose enrichment in plasma was determined by gas chromatography/mass spectrometry (6890 Network/5973 Series; Agilent, Santa Clara, CA, USA). Glucose clamps were performed 36-48 h after any bout of exercise to minimise the acute effects of exercise on insulin sensitivity. All participants were instructed to follow similar pre- and post-intervention diets for 3 days prior to the glucose clamp.

Blood chemistry

Fasting blood samples were obtained and processed. Plasma and serum were stored at $-70^{\circ} \mathrm{C}$ until assays were performed. Fasting glucose was measured by the glucose oxidase method (2300 Stat Plus; Yellow Springs Instruments, Yellow Springs, OH, USA). Fasting and clamp insulin were measured by ELISA (Millipore, Billerica, MA, USA). 
Body composition and maximal aerobic capacity

Total body fat and lean mass were assessed using dualenergy X-ray absorptiometry (Lunar, GE Lunar Prodigy and Encore 2005 software v9.30). A test for maximal aerobic capacity [10] was employed on a cycle ergometer before and after the intervention to determine changes in physical fitness and to determine the appropriate intensity for exercise prescription for those enrolled in EX.

Skeletal muscle biopsy and tissue analysis

Biopsy Approximately 30 min prior to the glucose clamp, a fasting percutaneous muscle biopsy was performed [8]. From the total sample $(\sim 100-300 \mathrm{mg})$, a portion was prepared for histochemical analysis as previously described by our group [9] and 30 mg flash frozen for lipid (DAG and ceramide) analysis.

Tissue analysis IMCL content was assessed using Oil Red O staining [6]. Succinate dehydrogenase (SDH) staining [10] was used as a marker of oxidative capacity. The percentages of type I, slow oxidative, and type IIa, glycolytic, fibres were determined using immunohistochemistry [10] with antibodies specific to the myosin heavy chain isoforms (Santa Cruz Biotechnology, Santa Cruz, CA, USA). Glycogen content was determined using a periodic acid-Schiff's reagent staining. DAG species, individual species of ceramide and selective sphingolipids were quantified using tandem mass spectrometry [27]. Total DAG and ceramide content was calculated by the addition of the individual species that represented $>90 \%$ (DAG, C16:0/16:0, C16:0/18:0, C16:0/18:1, C18:0/18:0, C18:0/ 18:1 and C18:1/18:1; ceramide, C16:0, C18:0, C24:0 and $\mathrm{C} 24: 1)$ of the identifiable lipid species, as a few lipid species were consistently at levels below detectable or quantifiable limits (DAG, C14:0/14:0, C14:0/16:0, C14:018:0, C14:018:1, C16:1/18:0, C16:1/18:1 and C16:1/16:1; ceramide, C14:0 and C20:0). Pre- and postintervention muscle samples from the same individual were analysed in parallel to avoid potentially confounding interassay variability influence on the intervention effects.

Protein extracts were prepared from $\sim 30-40 \mathrm{mg}$ skeletal muscle. Total SCD1 and DGAT1 levels were determined using standard immunoblot protocols. Briefly, liquid nitrogen crushed tissue was homogenised in cell lysis buffer (Cell Signaling, Boston, MA, USA) with protease inhibitors (Roche, Indianapolis, IN, USA). Samples were rocked for $1 \mathrm{~h}$ at $4^{\circ} \mathrm{C}$ and centrifuged for $20 \mathrm{~min}$ at $14,000 \mathrm{~g}$. Total protein content was determined by BSA and extracts prepared in $5 \times$ Laemmli buffer to provide $30-50 \mu \mathrm{g}$ protein. Samples were loaded on $10 \%$ SDS Ready Gels (BioRad, Hercules, CA, USA), transferred to immunoblot
PVDF membranes and blocked with $5 \%$ non-fat milk in PBS-Tween-20 (0.05\% vol./vol.). Protein samples for analysis for SCD1 (Alpha Diagnostics, San Antonio, TX, USA) and DGAT1 (Abcam, Cambridge, MA, USA) were explored with appropriate secondary antibodies following the manufacturers' recommendations. Blots were visualised using Immun-Star WesternC chemiluminescence (BioRad) and imaged with ChemiDoc XRS+ (BioRad). Densitometry was completed using ImageJ software. The control protein was $\beta$-actin.

\section{Statistical analysis}

Baseline group differences were assessed using a one-way ANOVA. A two-way repeated-measures ANOVA was used to determine main (group, treatment) and interaction effects for all outcome variables. Log transformations were used when ANOVA assumptions of normality were not met. Paired $t$ tests were used to examine intervention effects when repeated-measured ANOVA indicated an interaction effect. Pearson's correlation coefficient was used to relate alterations in muscle lipids to changes in insulin sensitivity. Statistical significance was assumed a priori at $p<0.05$. Statistical analyses were performed using the Statistical Package for the Social Sciences (SPSS for Mac, v18).

\section{Results}

\section{Study participants}

Approximately equal numbers of men and women were included in each intervention group (Table 1). All but four individuals had either impaired fasting glucose or IGT. Baseline $2 \mathrm{~h}$ OGTT values were not different between intervention groups.

\section{Body composition and physical fitness}

There were no baseline differences in body weight or composition between the intervention groups (Table 1). Each intervention decreased $(p<0.05)$ body weight, BMI and fat mass (Table 1). DIWL reduced body weight $(-8.5 \pm$ $1.5 \%)$ to a significantly greater extent $(p<0.05)$ than EX $(-1.8 \pm 0.9 \%)$. A similar pattern was observed in fat mass. Fat-free mass (FFM) was significantly decreased with DIWL $(-3.9 \pm 0.8 \%)$ and increased slightly in EX $(1.0 \pm$ $0.7 \%, p<0.05)$.

Baseline aerobic fitness levels (Table 1) were similar among the groups. Aerobic capacity tended to increase with EX $(p=0.07)$, but not with DIWL $(p=0.20$; Table 1$)$. Maximal power output was higher following EX $(93.6 \pm 6.6$ 
Table 1 Participant characteristics, body composition, glucose homeostasis and fitness

\begin{tabular}{|c|c|c|c|c|}
\hline \multirow[t]{2}{*}{ Characteristic } & \multicolumn{2}{|l|}{ DIWL } & \multicolumn{2}{|l|}{ EX } \\
\hline & Pre-intervention & Post-intervention & Pre-intervention & Post-intervention \\
\hline$n$ (men/women) & $8(3 / 5)$ & & $8(4 / 4)$ & \\
\hline Age (years) & $66.9 \pm 1.7$ & & $68.4 \pm 1.5$ & \\
\hline Body weight (kg) & $86.3 \pm 3.5$ & $78.9 \pm 3.1 *$ & $84.4 \pm 2.8$ & $82.7 \pm 2.3 *^{\dagger}$ \\
\hline BMI $\left(\mathrm{kg} / \mathrm{m}^{2}\right)$ & $31.2 \pm 1.2$ & $28.4 \pm 1.2 *$ & $30.0 \pm 1.0$ & $29.5 \pm 0.9 *^{\dagger}$ \\
\hline Fat mass $(\mathrm{kg})$ & $37.4 \pm 2.1$ & $32.1 \pm 2.4 *$ & $33.5 \pm 3.1$ & $31.8 \pm 2.9^{* \dagger}$ \\
\hline FFM (kg) & $45.2 \pm 2.8$ & $43.4 \pm 2.6$ & $47.4 \pm 2.4$ & $48.5 \pm 2.3^{\dagger}$ \\
\hline Fasting glucose (mmol/1) & $5.8 \pm 0.2$ & $5.4 \pm 0.1$ & $5.2 \pm 0.2$ & $5.3 \pm 0.1$ \\
\hline OGTT $2 \mathrm{~h}$ glucose $(\mathrm{mmol} / \mathrm{l})$ & $8.3 \pm 0.6$ & & $7.8 \pm 0.7$ & \\
\hline Fasting insulin (pmol/l) & $52.5 \pm 18.7$ & $30.8 \pm 8.8^{*}$ & $39.4 \pm 6.8$ & $39.7 \pm 6.1^{\dagger}$ \\
\hline$\dot{V} \mathrm{O}_{2 \max }\left(\mathrm{ml}\left[\mathrm{kg}_{\mathrm{FFM}}\right]^{-1} \min ^{-1}\right)$ & $30.7 \pm 2.1$ & $32.1 \pm 1.9$ & $31.5 \pm 2.1$ & $32.8 \pm 2.3$ \\
\hline
\end{tabular}

Data are mean \pm SEM

A two-way (group $\times$ time) repeated-measures ANOVA was used to assess differences

${ }^{*} p<0.05$ within-group intervention effect; ${ }^{\dagger} p<0.05$ between-groups difference

to $99.5 \pm 8.0 \mathrm{~W}, p=0.05)$, but not with DIWL $(94.0 \pm 6.1$ to $97.2 \pm 4.1 \mathrm{~W}, p=0.30$ ). The participants in the EX group adhered to the programme as evidenced by average exercise intensity $(72.9 \%$, range $65.0-86.8 \% ; 77.4 \%$ of maximal heart rate, range 70.0-85\%), confirmed from Polar heart rate recordings and reported exercise sessions per week (3.9 days, range 2.7-5.7 days).

Insulin sensitivity

Baseline insulin sensitivities were similar between groups. Insulin-stimulated glucose disposal was improved $(p<0.05)$ in DIWL $(20.6 \pm 4.8 \%)$ and EX $(19.2 \pm 12.9 \%)$ groups (Fig. 2). Insulin suppression of endogenous glucose production was improved with DIWL (-8.2 $\pm 4.1 \%$, $p=0.05)$, but not altered with EX $(-2.1 \pm 9.2 \%)$.

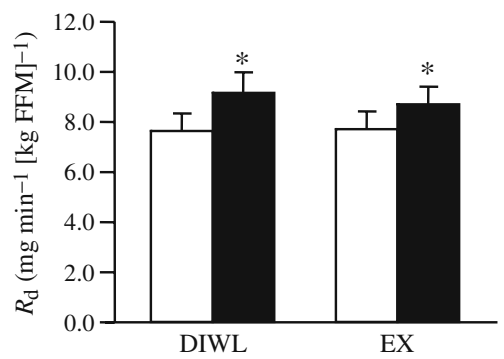

Fig. 2 Insulin sensitivity before (white bars) and after (black bars) a 16 week lifestyle intervention of DIWL $(n=8)$ or EX $(n=8)$. The rate of insulin-stimulated glucose disposal $\left(R_{\mathrm{d}}\right)$ was determined using as described in Methods and normalised to FFM. Data are mean \pm SEM. Group and intervention effects were explored using a two-way (group $\times$ time) repeated-measures ANOVA. ${ }^{*} p<0.05$ within-group intervention effect
Fasting glucose and insulin values were not different between the groups (Table 1). DIWL reduced fasting glucose $(-5.9 \pm 3.9 \%, p=0.07)$, while EX did not $(1.5 \pm 3.6 \%$, $p=0.40)$. Fasting insulin was reduced with DIWL $(-33.5 \pm$ $1.5 \%, p=0.04)$, but not with $\operatorname{EX}(10.4 \pm 10.7 \%, p=0.50)$. Steady-state insulin levels during the clamp were not different between the groups (DIWL, $373.1 \pm 21.6 \mathrm{pmol} / \mathrm{l}$ and EX, $337.8 \pm 23.1 \mathrm{pmol} / \mathrm{l}$ ) and were unchanged in response to the intervention.

Intramyocellular lipids, oxidative capacity and glycogen content

No baseline differences were observed in IMCL, SDH or glycogen content. Baseline differences in DAG species Di14:0 and Di-18:0, total ceramide content and ceramide species C20:0, C24:0 and C24:1 were observed. No other baseline differences were noted. Thus, subsequent analyses were weighted to the baseline measure.

DIWL reduced IMCL content $(-16.0 \pm 3.2 \%, p=0.01)$, while EX increased IMCL content $(40.8 \pm 18.2 \%, p=0.03$, Fig. 3a). SDH was decreased by DIWL $(-12.2 \pm 5.2 \%$, $p=0.04)$, but increased by $\mathrm{EX}(19.9 \pm 13.2 \%, p=0.05$, Fig. 3b). In order to examine whether changes in the proportion of type I muscle fibres, which contain more IMCL as triacylglycerols [23], influence changes in total IMCL content, we also examined changes in IMCL within specific fibre types. Although the changes in the proportion of type I slow-oxidative fibres were not statistically significant $(\mathrm{DIWL}=-4.9 \pm 8.1 \%$ and $\mathrm{EX}=6.5 \pm 7.5 \%), \mathrm{EX}$ tended ( $p=0.07, n=6)$ to increase IMCL content specific to type I fibres, while DIWL $(n=5)$ tended $(p=0.10)$ to 

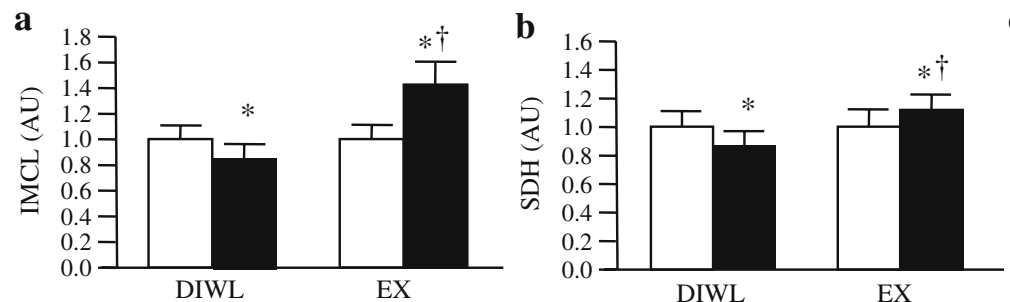

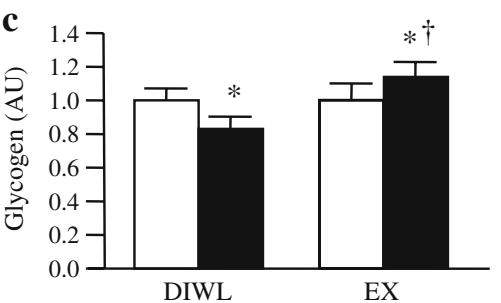

Fig. 3 Skeletal muscle substrate storage and capacity for oxidation before (white bars) and after (black bars) a 16 week lifestyle intervention of DIWL $(n=8)$ or EX $(n=8)$. All measures were performed on biopsy samples of the vastus lateralis. IMCL content, as determined by Oil Red O staining (a); SDH activity staining (b); and glycogen content (c) were determined using histochemical analyses as described in Methods. Data are mean \pm SEM. Group and intervention effects were explored using a two-way (group $\times$ time) repeated-measures ANOVA. ${ }^{*} p<0.05$ within-group intervention effect; ${ }^{\dagger} p<0.05$ between-groups difference decrease IMCL within type I fibres. Glycogen content (Fig. 3c) was decreased with DIWL $(-16.8 \pm 4.6 \%$, $p=0.04)$, but increased with EX $(13.9 \pm 3.3 \%, p=0.05)$.

We have recently published quantitative DAG and sphingolipid profiles for human muscle [23]. Much of the DAG data did not meet the ANOVA assumption of normality, thus the data were log transformed and analysed. Because the EX group did lose a minimal, albeit significant, amount of body weight, changes in body weight were used as a covariant in subsequent analyses. At baseline, no individual DAG species or total DAG content correlated with insulin sensitivity when all individuals were combined $(n=16)$. Both DIWL and EX decreased total DAG content (Fig. 4a). Both DIWL and EX decreased several of the DAG species, with EX decreasing C14:0/18:0, C16:1/18:0, C16:1/18:1, C18:0/18:0, DI-C16:1, DI-C18:0 and DI-C18:1 to a greater degree than DIWL (Table 2). Although the data did not reach statistical significance, EX tended $(p=0.10)$ to decrease total DAG to a greater degree than DIWL. (Absolute values for DAG species are shown in the electronic supplementary material [ESM] Table 1.)

The sphingolipid data did not meet ANOVA assumptions, thus the data were log transformed and analysed. Baseline total ceramide and several individual ceramide species were inversely $(p \leq 0.05)$ related to insulin sensitivity when all individuals were combined ( $n=16$, total ceramide $R=-0.54$, $\mathrm{C} 18: 0 R=-0.43, \mathrm{C} 18: 1 \quad R=-0.39, \mathrm{C} 20: 0 R=-0.45, \mathrm{C} 24: 0$ $R=-0.53$ and $\mathrm{C} 24: 1 \quad R=-0.43)$. No relation between other sphingolipids and insulin sensitivity was observed at baseline. Total ceramide content was decreased in EX $(p<0.05)$, but not with DIWL (Fig. 4b). All ceramide species were decreased with EX, while only C14:0, C20:0 and C24:0 ceramides were decreased with DIWL (Table 3). Dihydroceramide was decreased with both interventions (Table 3, Fig. 4c). Sphingosine was significantly decreased with EX $(p<0.05)$, but not DIWL (Table 3, Fig. 4d). Sphingosine 1-phosphate did not change (Fig. 4e). Absolute values for sphingolipid species are shown in ESM Table 2. Decreases in both C16:0 and C24:1 ceramide were signif- icantly ( $p \leq 0.05$ ) correlated with improved insulin-stimulated glucose disposal, while the change in total ceramide and other sphingolipids did not correlate to changes in insulin sensitivity.
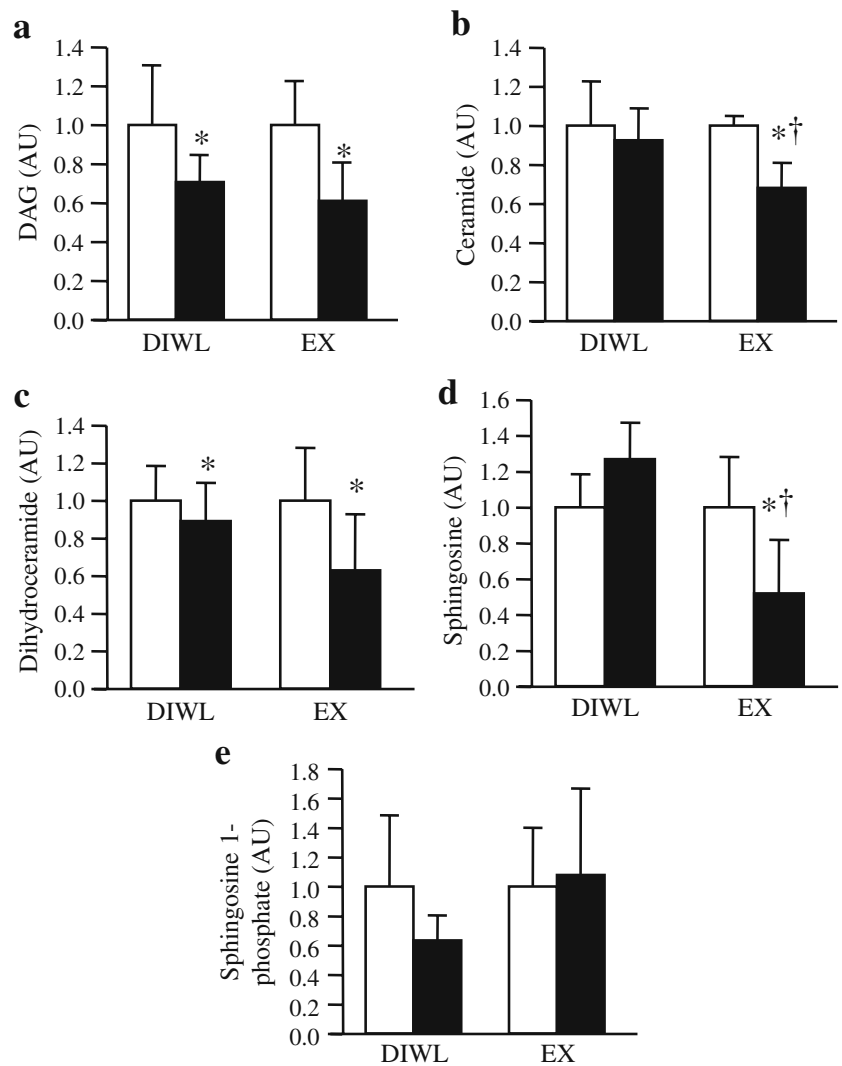

Fig. 4 Skeletal muscle DAG and ceramide before (white bars) and after (black bars) a 16 week lifestyle intervention of DIWL $(n=8)$ or EX $(n=8)$. Data for DAG (a), ceramide (b), dihydroceramide (c), sphingosine (d) and sphingosine 1-phosphate (e) were analysed using tandem mass spectrometry as described in Methods. Data are normalised to the 'pre' intervention values of each group. Group and intervention effects were explored using a two-way (group $\times$ time) repeated-measures ANOVA. Data were log transformed when ANOVA assumption of normality was not met. ${ }^{*} p<0.05$ withingroup intervention effect; ${ }^{\dagger} p<0.05$ between-groups difference 
Table 2 Percentage changes in individual and total diacylglycerol by intervention group

\begin{tabular}{|c|c|c|}
\hline DAG species & DIWL $^{\mathrm{a}}$ & $\mathrm{EX}^{\mathrm{b}}$ \\
\hline C14:0/16:0 & $-31.6 \pm 12.6^{*}$ & $-41.3 \pm 16.0 *$ \\
\hline C14:0/18:0 & $-17.1 \pm 20.9 *$ & $-42.9 \pm 12.7 *$ \\
\hline C14:0/18:1 & $-22.2 \pm 13.1^{*}$ & $-57.6 \pm 12.9 *$ \\
\hline C16:0/18:0 & $-21.4 \pm 10.6^{*}$ & $-34.2 \pm 12.7 *$ \\
\hline C16:0/18:1 & $-8.5 \pm 13.1^{*}$ & $-51.8 \pm 11.1^{*}$ \\
\hline C16:1/18:0 & $-16.9 \pm 11.1$ & $-63.9 \pm 8.9 * \dagger$ \\
\hline C16:1/18:1 & $-27.8 \pm 14.4 *$ & $-66.5 \pm 10.7 * \dagger$ \\
\hline C18:0/18:1 & $-15.3 \pm 5.8^{*}$ & $-57.8 \pm 9.9 * \dagger$ \\
\hline DI-C14:0 & $-41.2 \pm 8.6^{*}$ & $-58.1 \pm 10.9 *$ \\
\hline DI-C16:0 & $-26.7 \pm 10.8 *$ & $-52.9 \pm 14.7 *$ \\
\hline DI-C16:1 & $-41.3 \pm 15.3$ & $-55.0 \pm 16.2 * \dagger$ \\
\hline DI-C18:0 & $-8.8 \pm 18.4$ & $-48.4 \pm 13.9 * \dagger$ \\
\hline DI-C18:1 & $-22.8 \pm 8.8 *$ & $-64.1 \pm 11.2^{* \dagger}$ \\
\hline Total DAG & $-12.4 \pm 14.7 *$ & $-40.9 \pm 12.0 *$ \\
\hline
\end{tabular}

Data are mean \pm SEM

A two-way (group $\times$ time) repeated-measures ANOVA was used to assess differences

Data were log-transformed when ANOVA assumption of normality was not met

${ }^{\mathrm{a}} n=8$; three men/five women; ${ }^{\mathrm{b}} n=8$; four men/four women

${ }^{*} p<0.05$ within-group intervention; ${ }^{\dagger} p<0.05$ between-groups difference

Table 3 Percentage changes in individual and total ceramide and other sphingolipids by intervention group

\begin{tabular}{lcl}
\hline Species & DIWL $^{\mathrm{a}}$ & $\mathrm{EX}^{\mathrm{b}}$ \\
\hline Ceramide & & \\
C14:0 & $-14.8 \pm 19.3^{*}$ & $-33.1 \pm 15.2^{*}$ \\
C16:0 & $40.6 \pm 26.1$ & $-46.4 \pm 18.8$ \\
C18:0 & $20.8 \pm 19.2$ & $-45.1 \pm 12.3^{* \dagger}$ \\
C18:1 & $37.9 \pm 20.8$ & $-44.1 \pm 13.6$ \\
C20:0 & $-7.4 \pm 19.6^{*}$ & $-44.7 \pm 12.8^{*}$ \\
C24:0 & $-20.1 \pm 12.6^{*}$ & $-40.3 \pm 11.1^{*}$ \\
C24:1 & $15.1 \pm 19.3^{*}$ & $-44.3 \pm 12.3^{*}$ \\
Total & $1.6 \pm 13.6$ & $-36.1 \pm 11.8^{* \dagger}$ \\
Sphingolipids & & \\
Dihydroceramide & $-11.8 \pm 17.4^{*}$ & $-44.0 \pm 11.3^{*}$ \\
Sphingosine & $135.2 \pm 110.0$ & $-19.1 \pm 16.1^{* \dagger}$ \\
Sphingosine 1-phosphate & $36.7 \pm 38.0$ & $-28.1 \pm 30.5$ \\
\hline
\end{tabular}

Data are mean \pm SEM

A two-way (group $\times$ time) repeated-measures ANOVA was used to assess differences

Data were log transformed when ANOVA assumption of normality was not met

${ }^{\mathrm{a}} n=8$; three men/five women; ${ }^{\mathrm{b}} n=8$; four men/four women

${ }^{*} p<0.05$ within-group intervention; ${ }^{\dagger} p<0.05$ between-groups difference
Protein production

No baseline differences were observed in the production of SCD1 or DGAT1. No relation between production of SCD1 or DGAT1 and insulin sensitivity was observed. SCD1 production was significantly decreased $(-19.5 \pm 8.5$, $p=0.01)$ with DIWL, but increased with EX (19.6 \pm 7.4 , Fig. 5a). No differences were observed in production of DGAT1 (Fig. 5b). The change in production of SCD1 and DGAT1 did not predict improvements in insulin sensitivity. Change in SCD1 ( $\Delta$ vs $\Delta)$ was positively correlated with IMCL content $(R=0.56)$ and negatively correlated with ceramide $\mathrm{C} 16: 0(R=-0.64), \mathrm{C} 24: 0(R=-0.52)$ and total ceramide $(R=-0.55)$, all $p<0.05$. Change in DGAT1 was negatively correlated with ceramide $\mathrm{C} 20: 0 \quad(R=-0.81)$, $\mathrm{C} 24: 1(R=-0.76)$ and dihydroceramide $\mathrm{C} 16: 0(R=-0.82)$.

\section{Discussion}

Both DAG and ceramide have been implicated in the aetiology of insulin resistance. While cell-system and animal studies have demonstrated that these lipids can cause insulin resistance in particular situations [17, 19], studies in humans have only provided circumstantial, and contradictory, evidence regarding their potential role in

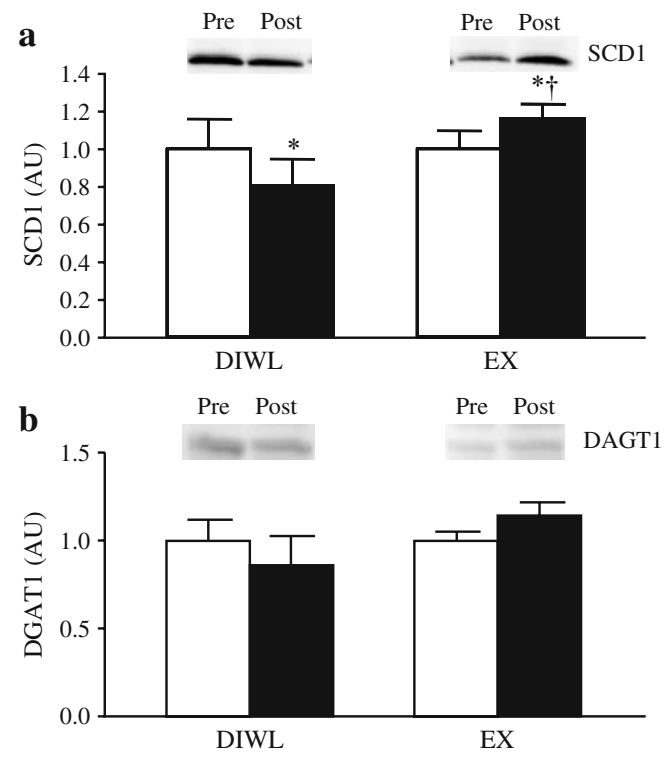

Fig. 5 Skeletal muscle SCD1 and DGAT1 before (white bars) and after (black bars) a 16-week lifestyle intervention of DIWL $(n=8)$ or EX $(n=8)$. Skeletal muscle protein production of SCD1 (a) and DGAT1 (b) were determined by western blot analysis as described in Methods. Data were normalised to the 'pre' intervention values of each group. Group and intervention effects were explored using a twoway (group $\times$ time) repeated-measures analysis of variance. ${ }^{*} p<0.05$ within-group intervention effect; ${ }^{\dagger} p<0.05$ between-groups difference 
skeletal muscle insulin resistance $[24,28]$. Therefore, the main purpose of this study was to examine whether or not changes in both the content and fatty acid profile of DAG, ceramide species and certain other sphingolipids correspond to improvements in insulin sensitivity with either DIWL or EX in humans.

The primary novel findings of our study were that both DIWL and exercise training decreased intramyocellular DAG content, while exercise decreased many individual subspecies and total ceramide content to a greater degree than DIWL. These decreases in DAG and ceramide with the interventions did not parallel changes in total IMCL content; DIWL decreased IMCL, while exercise training increased IMCL. Our study provides the first evidence concerning the separate effects of weight loss and exercise on these toxic lipids in human skeletal muscle, offering potential mechanisms by which lifestyle interventions may improve insulin sensitivity.

The decrease in skeletal muscle DAG by both exercise and weight loss corroborates a link between skeletal muscle DAG and insulin resistance [19, 29]. However, the lack of correlation between changes in DAG and improvements in insulin sensitivity suggests that DAG is not a sole mediator of insulin action [30]. Although we [9] and others [26] have previously reported exercise-induced decreases in DAG, this is the first study to directly compare the independent effects of weight loss and exercise on total and various species of DAG in human skeletal muscle. DAG was decreased with DIWL in parallel with IMCL, while exercise decreased DAG, but increased IMCL. These findings are supported by the observation that exercise decreases DAG $[9,26]$ but increases IMCL $[9,10]$. It is not clear why exercise induced greater reductions in specific subspecies of DAG than DIWL. Although the EX group did lose weight, the data do not support a combined effect of weight loss and exercise on IMCL or insulin sensitivity. DAG within skeletal muscle serves as a precursor for IMTG synthesis, is a substrate molecule, is a component of cell membranes and acts as a lipid-signalling second messenger [31]. Our data do not discern the subcellular localisation of DAG, its source or the specific roles it has within the cell. Nevertheless, our data suggest a repartitioning of NEFAs away from DAG, which is likely to be deleterious to insulin signalling, into neutral IMCL stores with exercise training or a decrease in total lipid content (i.e. DAG and IMCL) with DIWL.

Another key finding in our study was that exercise, but not DIWL, significantly decreased ceramide content within muscle concomitant with improved insulin sensitivity. This is in agreement previous observations $[9,26]$ that exercise training decreases skeletal muscle ceramide content. These data suggest that exercise training may decrease ceramide content through a shift in fatty acid partitioning away from de novo ceramide synthesis towards triacylglycerol formation and/or increased fatty acid oxidation within muscle. The lack of significant correlations between changes in ceramide and improved insulin sensitivity suggests that alterations in ceramide, as with DAG, are not solely required to improve insulin action. The quantification of ceramide subspecies and other sphingolipids is another novel aspect of this study. We observed that exercise resulted in significant decreases in both dihydroceramide and sphingosine. As dihydroceramide may not be as bioactive as ceramide and may only serve as a ceramide precursor [32], this decrease may not have a functional consequence. Alternatively, because sphingosine, an intermediate between ceramide and sphingosine 1-phosphate [32], activates PKC [33], increases in this lipid may impair insulin action. In fact, we observed significant positive correlations between changes in DAG C14:0/16:0, C14:0/18:1, C16:1/18:0, C16:1/18:1, DI-C14:0, DI-C16:1 and DI-C18:1 and changes in sphingosine in support of the sphingosine-DAG-PKC pathway. It is unclear why sphingosine or ceramide did not decrease with weight loss. Perhaps exercise induces additional or independent effects on insulin sensitivity compared with weight loss through reductions in ceramides or other sphingolipids within muscle. The effect of exercise, but not DIWL, to decrease specific sphingolipids and enhance the oxidative capacity in muscle is consistent with our report that ceramide content is associated with both insulin resistance and lower oxidative capacity of muscle [23].

Repartitioning of skeletal muscle lipids with lifestyle interventions concomitant with improved insulin sensitivity may be related to changes in key lipogenic enzymes. We report significant decreases in SCD1 with DIWL, but higher levels following exercise. This rate-limiting enzyme that converts saturated fatty acids to monounsaturated fatty acids has been implicated in the development of obesity [34]. Overproduction of SCD1 in muscle cells results in protection from lipid-induced insulin resistance $[14,35]$ and muscle protein production is increased following exercise training [13]. These studies, coupled with our observation of a significant correlation between SCD1 production and IMCL suggest that SCD1 is a key regulator of lipid partitioning within human skeletal muscle. Further, our data suggest that SCD1 is likely to contribute to decreases in several ceramide subspecies, supported by evidence of decreased ceramide content following acute exercise concomitant with increased IMCL and insulin sensitivity [13]. In accord with previous exercise studies [36, 37], we report no changes in production of DGAT1, which catalyses the final step in triacylglycerol synthesis. Increased DGAT1 production following acute exercise [13] suggests tightly coupled protein regulation in response to lipolytic stimuli. Finally, our observation of a negative correlation between DGAT1 and several ceramide species suggests an 
influence of DGAT1 in the repartitioning of fatty acids from deleterious lipid pools to more neutral IMCL stores.

Despite our novel observations there were study limitations. We did not have a control group; each participant acted as their own control in a randomised repeatedmeasures design. Low statistical power prevented a prospective sex-specific analysis. The exercise training stimulus may not have been sufficient to induce significant changes in fitness [38], yet still robust enough to elicit changes in oxidative capacity of muscle, IMCL and insulin sensitivity. The intervention effects on IMCL and insulin sensitivity were possibly mediated or modulated through changes in glycogen content or muscle fibre type, which were differentially affected by weight loss and exercise. The fatty acid composition of the diets may have affected the composition of DAGs or sphingolipids [39]. Thus, future studies are warranted to elucidate the potential compartmental differences in lipid intermediates, the role of individual DAG/sphingolipid subspecies, and optimal weight loss and/or exercise regimens to influence lipid partitioning within the context of improved insulin sensitivity in humans.

In summary, corresponding with improved insulin sensitivity, both DIWL and exercise decrease DAG content within skeletal muscle, while only exercise training appears to decrease ceramide and other sphingolipids. Despite similar improvements in insulin sensitivity, weight loss and exercise have opposing effects on IMTG and the oxidative capacity of skeletal muscle. Taken together, this study supports the concept that DAG and ceramide within skeletal muscle may be modifiable targets for interventions designed to improve insulin sensitivity in humans and, moreover, that lifestyle interventions repartition these deleterious lipids through alterations in fatty acid oxidation and triacylglycerol synthesis. However, the lack of correlation between alterations in these bioactive lipids and insulin sensitivity supports the concept of multiple causal pathways and subsequently multiple targets for improving insulin action in human muscle. Additional investigations are needed to address whether these DIWL- and exerciseinduced changes in specific species of these complex lipids underlie or are merely coincidental with improved insulin action.

Acknowledgements We thank the volunteers for participating and appreciate the efforts of the intervention staff (K. Clark, C. Fiedler, G. Grove, S. Anthony and the CTRC nurses).

Duality of interest The authors report no conflicts of interest. The study was supported by an ADA Clinical Research Award (B. H. Goodpaster), NIH R01 AG20128 (B. H. Goodpaster), Obesity and Nutrition Research (1P30DK46204) and Clinical and Translational Research Centers (UL1 RR024153).

\section{References}

1. Kim SH, Reaven GM (2008) Isolated impaired fasting glucose and peripheral insulin sensitivity: not a simple relationship. Diab Care 31:347-352

2. Muoio DM, Newgard CB (2006) Obesity-related derangements in metabolic regulation. Annu Rev Biochem 75:367-401

3. Bastie CC, Hajri T, Drover VA, Grimaldi PA, Abumrad NA (2004) CD36 in myocytes channels fatty acids to a lipaseaccessible triglyceride pool that is related to cell lipid and insulin responsiveness. Diabetes 53:2209-2216

4. Hegarty BD, Conney GJ, Kraegen EW, Furler SM (2002) Increased efficiency of fatty acid uptake contributes to lipid accumulation in skeletal muscle of high fat-fed insulin-resistant rats. Diabetes 51:1477-1484

5. Phillips DI, Caddy S, Ilic V et al (1996) Intramuscular triglyceride and muscle insulin sensitivity: evidence for a relationship in nondiabetic subjects. Metabolism 45:947-950

6. Goodpaster BH, Theriault R, Watkins SC, Kelley DE (2000) Intramuscular lipid content is increased in obesity and decreased by weight loss. Metabolism 49:467-472

7. Goodpaster BH, Kelley DE, Wing RR, Meier A, Thaete FL (1999) Effects of weight loss on regional fat distribution and insulin sensitivity in obesity. Diabetes 48:839-847

8. Goodpaster BH, He J, Watkins S, Kelley DE (2001) Skeletal muscle lipid content and insulin resistance: evidence for a paradox in endurance-trained athletes. J Clin Endocrinol Metab 86:5755-5761

9. Dube JJ, Amati F, Stefanovic-Racic M, Toledo FG, Sauers SE, Goodpaster BH (2008) Exercise-induced alterations in intramyocellular lipids and insulin resistance: the athlete's paradox revisited. Am J Physiol Endocrinol Metab 294:E882-E888

10. Pruchnic R, Katsiaras A, He J, Kelley DE, Winters C, Goodpaster BH (2004) Exercise training increases intramyocellular lipid and oxidative capacity in older adults. Am J Physiol Endocrinol Metab 287:E857-E862

11. van Loon LJ, Koopman R, Manders R, van der Weegen W, van Kranenburg GP, Keizer HA (2004) Intramyocellular lipid content in type 2 diabetes patients compared with overweight sedentary men and highly trained endurance athletes. Am J Physiol Endocrinol Metab 287:E558-E565

12. Tarnopolsky MA, Rennie CD, Robertshaw HA, FedakTarnopolsky SN, Devries MC, Hamadeh MJ (2007) Influence of endurance exercise training and sex on intramyocellular lipid and mitochondrial ultrastructure, substrate use, and mitochondrial enzyme activity. Am J Physiol Regul Integr Comp Physiol 292: R1271-R1278

13. Schenk S, Horowitz JF (2007) Acute exercise increases triglyceride synthesis in skeletal muscle and prevents fatty acid-induced insulin resistance. J Clin Invest 117:1690-1698

14. Peter A, Weigert C, Staiger H et al (2009) Individual stearoylCoA desaturase 1 expression modulates endoplasmic reticulum stress and inflammation in human myotubes and is associated with skeletal muscle lipid storage and insulin sensitivity in vivo. Diabetes 58:1757-1765

15. Smith IJ, Huffman KM, Durheim MT, Duscha BD, Kraus WE (2009) Sex-specific alterations in mRNA level of key lipid metabolism enzymes in skeletal muscle of overweight and obese subjects following endurance exercise. Physiol Genomics 36:149-157

16. Mayer-Davis EJ, D'Agostino R Jr, Karter AJ et al (1998) Intensity and amount of physical activity in relation to insulin sensitivity: the Insulin Resistance Atherosclerosis Study. JAMA 279:669-674

17. Chavez JA, Knotts TA, Wang L et al (2003) A role for ceramide, but not diacylglycerol, in the antagonism of insulin signal transduction by saturated fatty acids. J Biol Chem 278:10297-10303 
18. Adams JM 2nd, Pratipanawatr T, Berria R et al (2004) Ceramide content is increased in skeletal muscle from obese insulin-resistant humans. Diabetes 53:25-31

19. Yu C, Cline GW, Zhang D et al (2002) Mechanism by which fatty acids inhibit insulin activation of insulin receptor substrate-1 (IRS-1)-associated phosphatidylinositol 3-kinase activity in muscle. J Biol Chem 277:50230-50236

20. Summers SA (2006) Ceramides in insulin resistance and lipotoxicity. Prog Lipid Res 45:42-72

21. Hoy AJ, Brandon AE, Turner $\mathrm{N}$ et al (2009) Lipid and insulin infusion-induced skeletal muscle insulin resistance is likely due to metabolic feedback and not changes in IRS-1, Akt, or AS160 phosphorylation. Am J Physiol Endocrinol Metab 297:E67-E75

22. Moro C, Galgani JE, Luu L et al (2009) Influence of gender, obesity, and muscle lipase activity on intramyocellular lipids in sedentary individuals. J Clin Endocrinol Metab 94:3440-3447

23. Coen PM, Dube JJ, Amati F et al (2010) Insulin resistance is associated with higher intramyocellular triglycerides in type I but not type II myocytes concomitant with higher ceramide content. Diabetes 59:80-88

24. Skovbro M, Baranowski M, Skov-Jensen C et al (2008) Human skeletal muscle ceramide content is not a major factor in muscle insulin sensitivity. Diabetologia 51:1253-1260

25. Schmitz-Peiffer C, Craig DL, Biden TJ (1999) Ceramide generation is sufficient to account for the inhibition of the insulin-stimulated PKB pathway in $\mathrm{C} 2 \mathrm{C} 12$ skeletal muscle cells pretreated with palmitate. J Biol Chem 274:24202-24210

26. Bruce CR, Thrush AB, Mertz VA et al. (2006) Endurance training in obese humans improves glucose tolerance, mitochondrial fatty acid oxidation and alters muscle lipid content. Am J Physiol Endocrinol Metab 291:E99-E107

27. Bielawski J, Szulc ZM, Hannun YA, Bielawska A (2006) Simultaneous quantitative analysis of bioactive sphingolipids by high-performance liquid chromatography-tandem mass spectrometry. Methods 39:82-91
28. Straczkowski M, Kowalska I, Nikolajuk A et al (2004) Relationship between insulin sensitivity and sphingomyelin signaling pathway in human skeletal muscle. Diabetes 53:1215-1221

29. Itani SI, Rudderman NB, Schmieder F, Boden G (2002) Lipid-induced insulin resistance in human muscle is associated with changes in diacylglycerol, protein kinase $\mathrm{C}$ and IkB- $\alpha$. Diabetes 51:2005-2011

30. Erion DM, Shulman GI (2010) Diacylglycerol-mediated insulin resistance. Nat Med 16:400-402

31. Carrasco S, Merida I (2007) Diacylglycerol, when simplicity becomes complex. Trends Biochem Sci 32:27-36

32. Bartke N, Hannun YA (2009) Bioactive sphingolipids: metabolism and function. J Lipid Res 50(Suppl):S91-S96

33. Smith ER, Merrill AH, Obeid LM, Hannun YA (2000) Effects of sphingosine and other sphingolipids on protein kinase C. Methods Enzymol 312:361-373

34. Biddinger SB, Almind K, Miyazaki M, Kokkotou E, Ntambi JM, Kahn CR (2005) Effects of diet and genetic background on sterol regulatory element-binding protein-1c, stearoyl-CoA desaturase 1 , and the development of the metabolic syndrome. Diabetes 54:1314-1323

35. Pinnamaneni SK, Southgate RJ, Febbraio MA, Watt MJ (2006) Stearoyl CoA desaturase 1 is elevated in obesity but protects against fatty acid-induced skeletal muscle insulin resistance in vitro. Diabetologia 49:3027-3037

36. Schmitt B, Fluck M, Decombaz J et al (2003) Transcriptional adaptations of lipid metabolism in tibialis anterior muscle of endurance-trained athletes. Physiol Genomics 15:148-157

37. Alsted TJ, Nybo L, Schweiger M et al (2009) Adipose triglyceride lipase in human skeletal muscle is upregulated by exercise training. Am J Physiol Endocrinol Metab 296:E445-E453

38. Bouchard C, An P, Rice T et al (1999) Familial aggregation of VO (2max) response to exercise training: results from the HERITAGE Family Study. J Appl Physiol 87:1003-1008

39. Ichi I, Nakahara K, Kiso K, Kojo S (2007) Effect of dietary cholesterol and high fat on ceramide concentration in rat tissues. Nutrition 23:570-574 